\title{
Tight-Binding Analysis of Electronic Structure of Germanene Sheet and Nanoribbons Including Stone-Wales Defect
}

\section{Komeil Rahmani}

Semnan University

Saeed Mohammadi ( $\nabla$ sd.mohammadi@semnan.ac.ir)

Semnan university https://orcid.org/0000-0002-3143-1650

\section{Research Article}

Keywords: Germanene, Stone-Wales defect, Tight binding method, Nanoribbon, Energy band structure

Posted Date: May 17th, 2021

DOl: https://doi.org/10.21203/rs.3.rs-462442/v1

License: (c) (1) This work is licensed under a Creative Commons Attribution 4.0 International License.

Read Full License 


\title{
Tight-binding Analysis of Electronic Structure of Germanene Sheet and Nanoribbons Including Stone-Wales Defect
}

\author{
Komeil Rahmani ${ }^{1}$, Saeed Mohammadi ${ }^{1 *}$ \\ ${ }^{1}$ Department of Electrical Engineering, Semnan University, Semnan, Iran \\ "Correspondence should be addressed to Saeed Mohammadi: sd.mohammadi@ semnan.ac.ir.
}

\begin{abstract}
In this paper, we investigate the electronic characteristics of germanene using the tight binding approximation. Germanene as the germanium-based analogue of graphene has attracted much research interest in recent years. Our analysis is focused on the pristine sheet of germanene as well as defective monolayer. The StoneWales defect, which is one of the most common topological defects in such structures, is considered in this work. Not only the infinite sheet of germanene but also the germanene nanoribbons in different orientations are analyzed. The obtained results show that applying the Stone-Wales defect into the germanene monolayer changes the energy band structure; the E-k curves around the Dirac point are no longer linear, a band gap is opened, and the Fermi velocity is reduced to half of that of defect-free germanene. In the case of nanoribbon structures, the armchair germanene nanoribbons with nanoribbon widths of $3 p$ and $3 p+1$ reveal the semiconductor behaviour. However, armchair germanene nanoribbon with width of $3 p+2$ is semi-metal. After applying the Stone-Wales defect, the band gap of armchair germanene nanoribbons with widths of $3 p$ and $3 p+1$ is reduced and it is increased for the width of $3 p+2$. Furthermore, there is no band gap in the energy band structure of zigzag germanene nanoribbon and the metallic behaviour is obvious.
\end{abstract}

Keywords: Germanene, Stone-Wales defect, Tight binding method, Nanoribbon, Energy band structure.

\section{Introduction}

Germanium and carbon are isoelectronic atoms. Carbon in bulk form is more commonly found in the hexagonal phase with $s p^{2}$ hybridization, whereas germanium in the cubic phase is more stable with $s p^{3}$ hybridization. Germanium with a large ionic radius promotes $s p^{3}$ hybridization. In germanene (a monolayer of germanium atoms) the mixture of $s p^{2}$ and $s p^{3}$ hybridization causes a prominent buckling distance of $0.68 \AA[1,2]$. Although bulk germanium is a significant indirect-bandgap semiconductor with excellent compatibility with silicon technology, but germanene, similar to graphene, shows linear dispersion relation around the $K$ and $K^{\prime}$ points of the first Brillouin zone, in which the conduction band (CB) and the valence band (VB) touch each other [3, 4]. Owing to the spin-orbit interaction in germanene, it has massive Dirac fermion, unlike to massless fermions in graphene $[4,5]$. The effective electronic masses $\left(m^{*}\right)$ of graphene and germanene are 0 and $0.007 \mathrm{~m}_{0}$, respectively. Atom-atom bond length of germanene is $2.431 \AA$, whereas it is $1.422 \AA$ for graphene, and lattice constant of germanene and graphene are $4.043 \AA$ and $2.463 \AA$, respectively [2-5]. Since $\mathrm{Ge}-\mathrm{Ge}$ bonds of germanene are more flexible than $\mathrm{C}-\mathrm{C}$ ones of graphene, the 
elastic constant of germanene with the highest buckled structure and graphene with no buckled structure are $56.01\left(\mathrm{Jm}^{-2}\right)$ and $328.02\left(\mathrm{Jm}^{-2}\right)$, respectively [6]. According to the ab initio studies, germanene has two different buckled structures, known as low buckling (LB) and high buckling (HB). Germanene with LB geometry (with less than $1 \AA$ buckling) is stable and acts like a semi-metal. Furthermore, the buckled structure causes reduction of electron-phonon coupling strength [7]. The $\sigma$ and $\pi$ bonds between atoms of germanene with LB geometry are separately coupled and there is a weak $\pi$ - $\pi$ interaction among them [8]. Figure 1 indicates germanene as a honeycomb monolayer of Ge atoms.

(a)

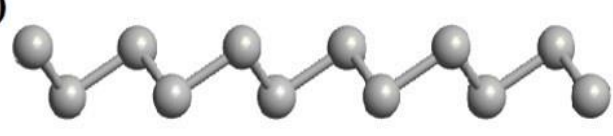

(b)

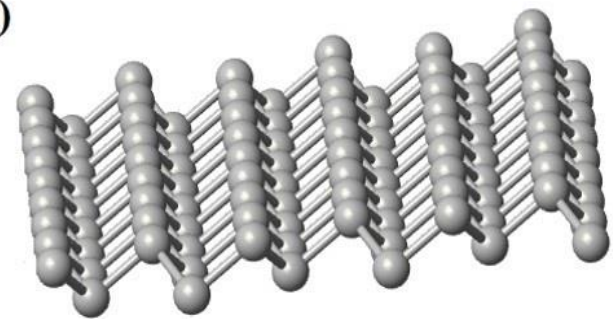

(c)

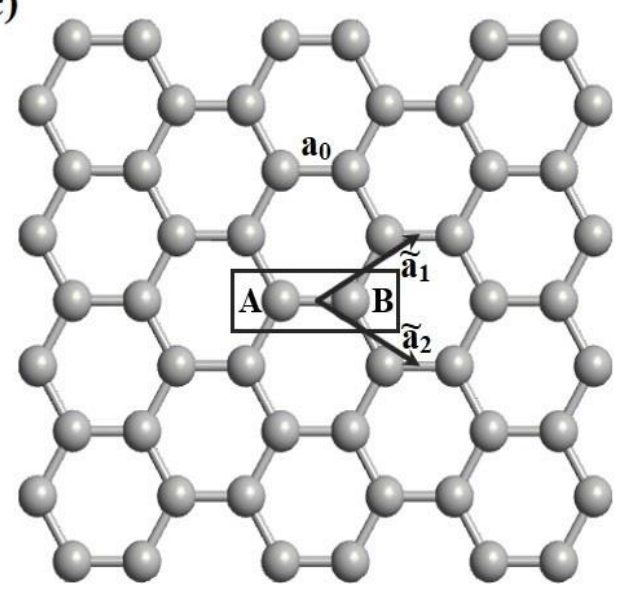

Figure 1. Structure of germanene in different views (a) and (b) side, and (c) top. $\tilde{a}_{1}$ and $\tilde{a}_{2}$ are primitive lattice vectors, and $\mathrm{a}_{0}$ is the Ge-Ge bond length. The unit cell includes two atoms, named $\mathrm{A}$ and $\mathrm{B}$.

The value of Fermi velocity in germanene is $30 \%$ lower compared to that of graphene, however, interactions of carrier with phonons in germanene are 25 times less than those in graphene which indicate its high mobility of carriers [7]. The mobility of electrons in graphene is about $3.39 \times 10^{5} \mathrm{~cm}^{2} V^{-1} \mathrm{~s}^{-1}$, while that is calculated about $6.09 \times 10^{5} \mathrm{~cm}^{2} V^{-1} \mathrm{~s}^{-1}$ for germanene sheet. The high carrier mobility of germanene is also attributed to the small electron effective mass and the large buckled distance [6]. Germanene benefits from strong molecular adsorption and high surface reactivity features which may be utilized for gas sensing applications or band gap engineering [9]. Germanene sheet also has considerable light absorption of solar spectrum and demonstrates optical anisotropy. Study of the phonon spectrum has demonstrated that the lattice of germanene is stable against up to $16 \%$ strain and the Dirac cone shifts towards higher energies when the strain is increased [4]. 
Owing to the buckling, it is easier to open a band gap in germanene than in the planar graphene. Although germanene is a zero-gap semi-metallic, however by applying periodic nanoholes, chemisorption of adatom species, lateral confinement, hydrogenation, introducing uniaxial and biaxial strain, edge functionalization, doping with different impurities, organic molecule adsorption, single-side adsorption of alkali metal (AM) atoms, coupling with a substrate, applying vertical electric field, and defects it is possible to open a band gap in germanene [8-12]. In [3] authors have reported the dependence of the energy band gap of armchair germanene nanoribbon (AGeNR) on width of ribbon and the shape of its edges.

The above mentioned prominent and diverse features of germanene make it as a promising candidate in many fields such as; application in spintronics and quantum computing due to robust quantum spin Hall effect, FETs with germanene channel, electronic and photonic devices, gas sensors and biosensing applications as a biomolecular recognition element [13-21]. The transport and electronic characteristics of germanene are effectively modified by chemical functionalization, doping, various shaped molecular junctions, and topological defects [22, 23]. The presence of defects produces scattering states close to the Fermi level, which diminishes the current flow in the monolayer [23]. The controllable defects in germanene involve the adatom, substitution, disorder, ripple, vacancy and Stone Wales (SW) defect. The SW defect is likely to be present and detectable in nanomaterial under nonequilibrium conditions, whereas formed through the effects of mechanical strain or application of low energy electron irradiation [23, 24]. It is very significant to investigate how structural defects, such as SW defect, can affect the band structural of germanene with lowest formation energy. After the creation of SW defect, germanene preserves its buckled structure with only a local out-of-plane displacement of the Ge atoms [23, 24]. Figure 2 shows the SW defect in germanene where an in-plane $90^{\circ}$ rotation of two Ge atoms regarding the midpoint of the Ge-Ge bond occurs and consequently, four neighboring hexagons change into two pentagons and two heptagons. The SW defects locally alter the Ge-Ge bond length and the natural angle between the two adjacent bonds. As it is shown in the figure, the length of Ge-Ge bond decreases approximately $2 \%$ from 2.460 to $2.407 \AA$ and increases approximately $7 \%$ to $2.642 \AA$ [23]. 


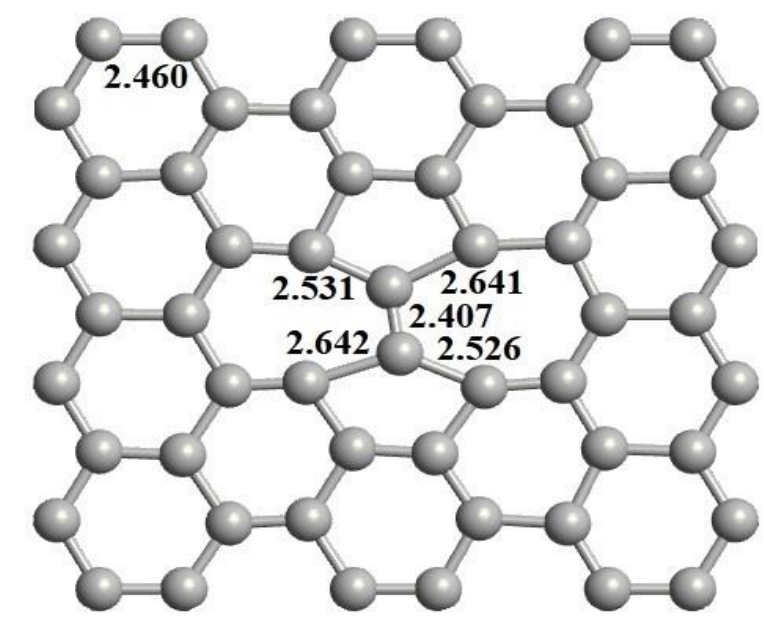

Figure 2. Structure of germanene monolayer with SW defect.

In recent decade, characterization of electronic behavior of defect-free and defective germanene has been studied by methods such as ab initio molecular dynamic calculations, Green's function approach, and first-principles density function theory calculations [14-18, 23]. In this paper, utilizing tight binding (TB) method, we investigate the energy band structure of pristine germanene and germanene with SW defect. The infinite sheet and nanoribbon of germanene in different directions are considered. This paper is organized as follows: in section II, the TB method and the generalized TB formalism are presented. The obtained results and main findings are shown in section III, and concluding remarks are given in section IV.

\section{Tight-Binding Method}

Among various approaches to determine the energy band structure of a material and study the effects of doping, defect, molecule adsorption, strain, external electric field, and light radiation on this structure, the TB method is the fastest and the simplest. It is a fully quantum mechanical approach to investigate the electronic characteristics of molecules and solids. TB calculations offer an attractively direct and transparent picture of chemical bonding. TB method is lower cost and spends much less computation time compared to the other more complicated methods, however, it can provide qualitatively and often quantitatively accurate results. In fact, the TB method lies between the very accurate and expensive ab initio methods and the fast but limited empirical approaches [25-30]. Since the system studied in this research is germanene and most of the unique properties of germanene are related to Dirac's 
points, Dirac's relativistic equations for the electron-hole can be calculated using the Taylor expansion of the firstorder Hamiltonian matrix in the TB method. By generalization the two-particle state of the electron-hole, Hamiltonian can be obtained. A special mapping transforms the Hamiltonian of system into a harmonic oscillator equation, in which the calculation of the eigenfunctions and eigenvalues of the system is possible. This method is very scalable, i.e. its computational complexity can be adjusted based on the aim of the study by selecting the number of basis sets and hopping neighbors. In TB method, the proper determination of related on-site and hopping energies is fundamentally significant for the correct representation of the band structure of material [25-30]. TB method is implemented here to model the electronic properties of germanene near the Fermi level, which can give precise results in the low-energy range. In this method, the required TB parameters such as the unit cell size, lattice coordinates, corresponding reciprocal lattice vectors, and the distance of each atom from its nearest neighbor atoms are considered. The buckling distance along the $\mathrm{z}$ direction and its effect on germanene band structure is considered in TB calculations. There is a relationship between the energy bands of germanene and buckling distance.

In Figure 1 we also illustrate the unit cell and the primitive lattice vectors ( $\tilde{a}_{1}$ and $\tilde{a}_{2}$, where $\mathrm{a}_{0}$ is the Ge-Ge bond length) to be used in TB method. Unlike the equal applied forces to each Ge atom in pristine germanene, in defective germanene, atoms with closer distances interact more, thereby, it disturbs the energy levels in the defective germanene and results in nonlinearity of the diagram near the Dirac point. The generalized TB formalism includes the following equations (1-10). The unit cell consists of two germanium atoms, named $\mathrm{A}$ and $\mathrm{B}$, with frontier atomic wave functions of $\varphi_{A}$ and $\varphi_{B}$, respectively. The wave function of the unit cell may be obtained by the linear combination of the atomic states,

$\varphi(r)=c_{1} \varphi_{A}(r)+c_{2} \varphi_{B}(r)$.

The TB wave function is given by,

$\psi(r)=\frac{1}{\sqrt{N}} \sum_{R} e^{i k R} \varphi(r-R)$

where $N$ is the number of unit cells in the crystal, and $R$ is the position of the unit cell. The band structure can be obtained by solving the Schrodinger equation as,

$H|\psi(r)\rangle=E(k)|\psi(r)\rangle$

where $H$ and $E(k)$ are the Hamiltonian matrix and eigenvalue, respectively. We multiply the crystal Schrodinger equation by the atomic wave functions as follow, 
$\left\langle\varphi_{A}(r)|H| \psi(r)\right\rangle=E(k)\left\langle\varphi_{A} \mid \psi(r)\right\rangle$

$\left\langle\varphi_{B}(r)|H| \psi(r)\right\rangle=E(k)\left\langle\varphi_{B} \mid \psi(r)\right\rangle$

Considering only interactions between the frontier atomic orbitals of nearest neighbors, the tight binding matrix is obtained. The dispersion relation of the structure is obtained from the following relation, which is achieved when we look for nontrivial solutions for the weighting factors, $c_{1}$ and $c_{2}$,

$|\mathrm{H}-E S|=0$

where

$\mathrm{H}=\left[\begin{array}{ll}H_{A A} & H_{A B} \\ H_{B A} & H_{B B}\end{array}\right]$ and $S=\left[\begin{array}{ll}S_{A A} & S_{A B} \\ S_{B A} & S_{B B}\end{array}\right]$ are Hamiltonian and overlap matrices, respectively. The elements of the Hamiltonian matrix can be determined as follows,

$H_{X X}=\frac{1}{N} \sum_{i=1}^{N}\left\langle\varphi_{X}\left(r-R_{X, i}\right)|H| \varphi_{X}\left(r-R_{X, i}\right)\right\rangle ; \quad X=A, B$

where $E_{2 p}=\left\langle\varphi_{A}\left(r-R_{A, i}\right)|H| \varphi_{A}\left(r-R_{A, i}\right)\right\rangle$ is the energy of the $2 p_{z}$ orbital. The hopping interactions between the A and $\mathrm{B}$ atoms, including all the $\mathrm{A}$ sites positioned at $R_{A, i}$ and all the $\mathrm{B}$ sites positioned at $R_{B, j}$, are determined by

$H_{A B}=\frac{1}{N} \sum_{i=1}^{N} \sum_{j=1}^{N} e^{i k\left(R_{B, j}-R_{A, i}\right)}\left\langle\varphi_{A}\left(r-R_{A, i}\right)|H| \varphi_{B}\left(r-R_{B, j}\right)\right\rangle$.

Moreover, the overlap matrix elements are determined as follows,

$S_{A B}=\frac{1}{N} \sum_{i=1}^{N} \sum_{j=1}^{N} e^{i k\left(R_{B, j}-R_{A, i}\right)}\left\langle\varphi_{A}\left(r-R_{A, i}\right) \mid \varphi_{B}\left(r-R_{B, j}\right)\right\rangle$.

It should be noted that $S_{A A}=S_{B B}=1$. For an individual A atom, there are three nearest neighbors of B atoms, so $H_{A B}$ can be approximated as,

$H_{A B} \approx \gamma_{0} \sum_{l=1}^{3} e^{i k d_{l}} ; \quad$ where $\quad \gamma_{0}=\left\langle\varphi_{A}\left(r-R_{A, i}\right)|H| \varphi_{B}\left(r-R_{B, j}\right)\right\rangle$

and $d_{l}$ is the distance between the neighbors. The overlap matrix elements can be approximated too,

$S_{A B} \approx S_{0} \sum_{l=1}^{3} e^{i k d_{l}} ; \quad \quad$ where $\quad s_{0}=\left\langle\varphi_{A}\left(r-R_{A, i}\right) \mid \varphi_{B}\left(r-R_{B, j}\right)\right\rangle$.

Since two sublattices are equivalent, $H_{A A}=H_{B B}, S_{A A}=S_{B B}, H_{B A}=H_{A B}^{*}$, and $S_{B A}=S_{A B}^{*}$. 


\section{Results and Discussion}

\section{- The energy band structure of infinite germanene sheet}

The energy band structure of infinite germanene is depicted in figure 3(a). The absence of band gap and linear dispersion relation near the zero energy level is observed in the figure. The Fermi velocity at Dirac point is about $5.6 \times 10^{5} \mathrm{~ms}^{-1}$, which is related to the slope of E-k curves around this point [7]. As shown in figure 3(b), after applying the Stone-Wales defect to the infinite germanene, the E-k curves are no longer linear and a band gap is opened. In this case, the value of Fermi velocity is $2.75 \times 10^{5} \mathrm{~ms}^{-1}$, which is about $50 \%$ less than that of defect-free germanene. In the other words, due to a decrease in the slope of the curves around the Dirac point, the Fermi velocity reduces. In addition, the energy band structure of defective germanene has a direct band gap of about $0.07 \mathrm{eV}$. The results indicate a good agreement with the published data in [23], where authors employed ab initio calculations to investigate the same structure.

(a)

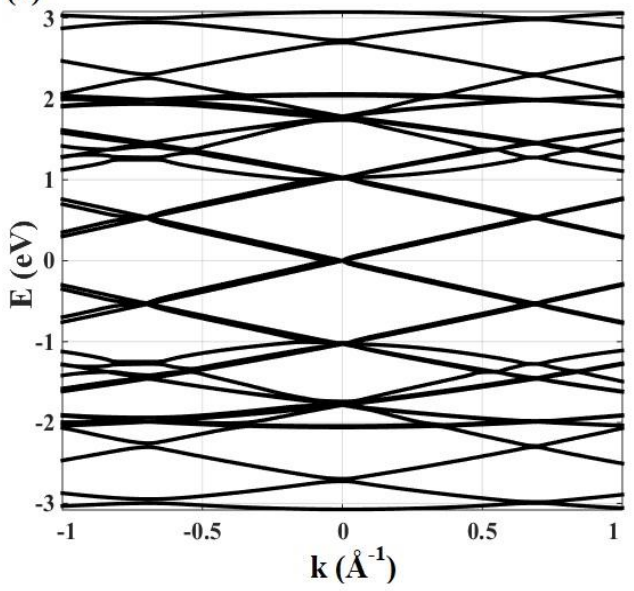

(b)

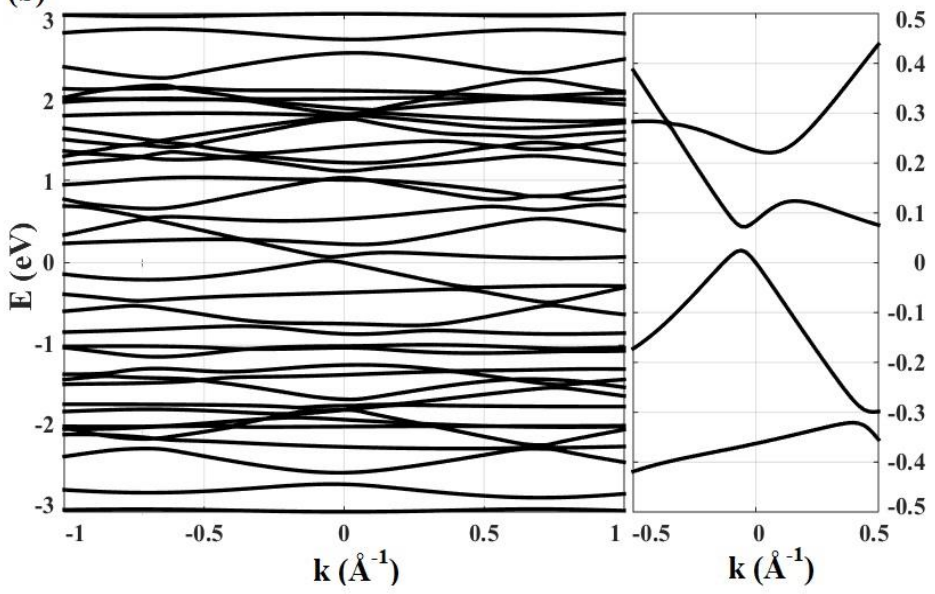

Figure 3. Energy band structure of (a) defect-free, and (b) defective infinite germanene sheet.

\section{- The armchair germanene nanoribbon (AGeNR) energy band structure}

Figure 4 shows the unit cell in AGeNR with different nanoribbon widths $(N=3 p, 3 p+1$ and $3 p+2)$, in which $N$ is the number of germanene dimer lines and $p$ is a positive integer. AGeNRs with widths of $3 p$ and $3 p+1$ show the semiconducting behavior, while AGeNR with width of $3 p+2$ is semi-metal, as depicted in figure 5 . The direct band gaps of defect-free AGeNRs with $N=3 p, 3 p+1$ and $3 p+2$ are about $0.36 e V, 0.39 e V$ and $0.009 e V$, respectively. The 
obtained results for defect-free AGeNR are in close match with the reported data in [3], where DFT method was employed to obtain the electronic properties of the same structures.

(a)

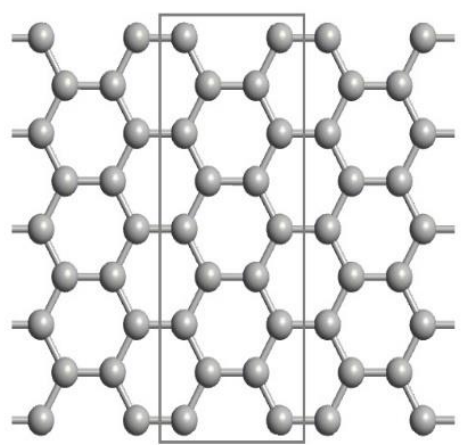

(b)

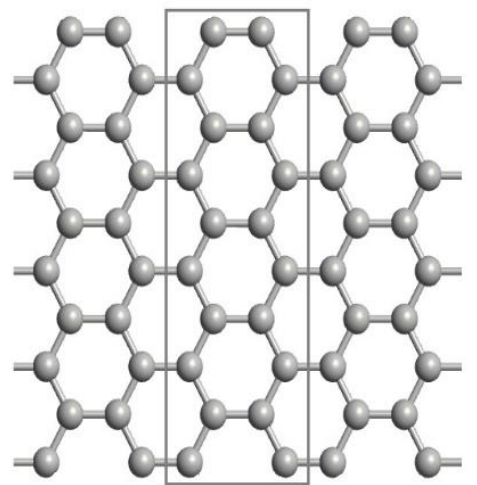

(c)

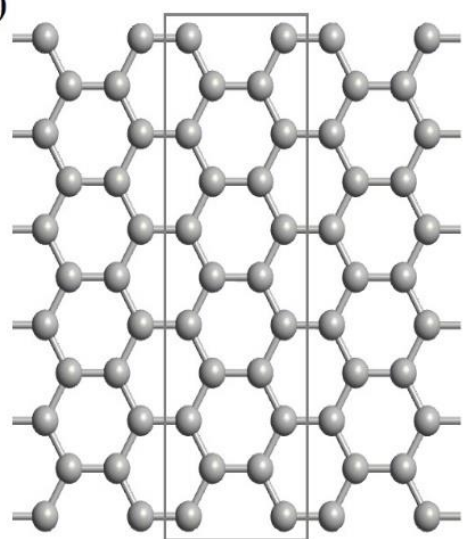

Figure 4. AGeNR with different nanoribbon widths, (a) $3 p$, (b) $3 p+1$, (c) $3 p+2$.

The dispersion relations of defective AGeNR with different widths are shown in figure 6 . As it can be inferred from the figure, the SW defect modulates the band structure of the nanoribbons of different widths. The band gaps of defective AGeNRs with $N=3 p, 3 p+1$ and $3 p+2$ are about $0.12 e V, 0.38 \mathrm{eV}$ and $0.24 \mathrm{eV}$, respectively. In all cases the semiconducting behavior of the structures are clearly evident in the figures. The results demonstrate that the band gap of AGeNR with width of $3 p+1$ is the largest, moreover, applying the SW defect to AGeNR with width of $3 p+2$ converts this semi-metal sheet to a semiconductor and leads to a decrease in the Fermi velocity near the Dirac point. 


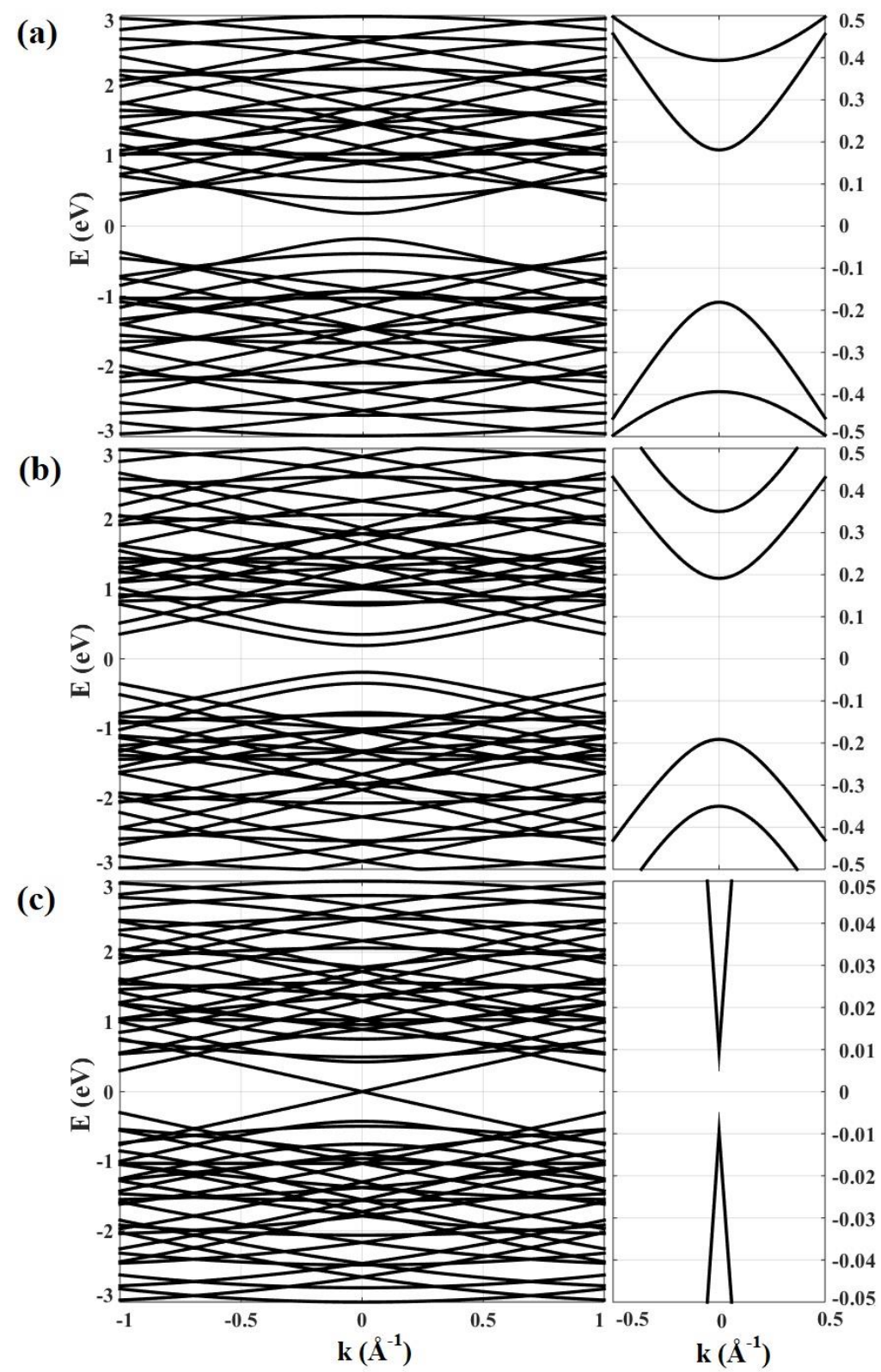

Figure 5. Energy band structure of defect-free AGeNR with (a) $N=3 p$, (b) $N=3 p+1$, (c) $N=3 p+2$. 


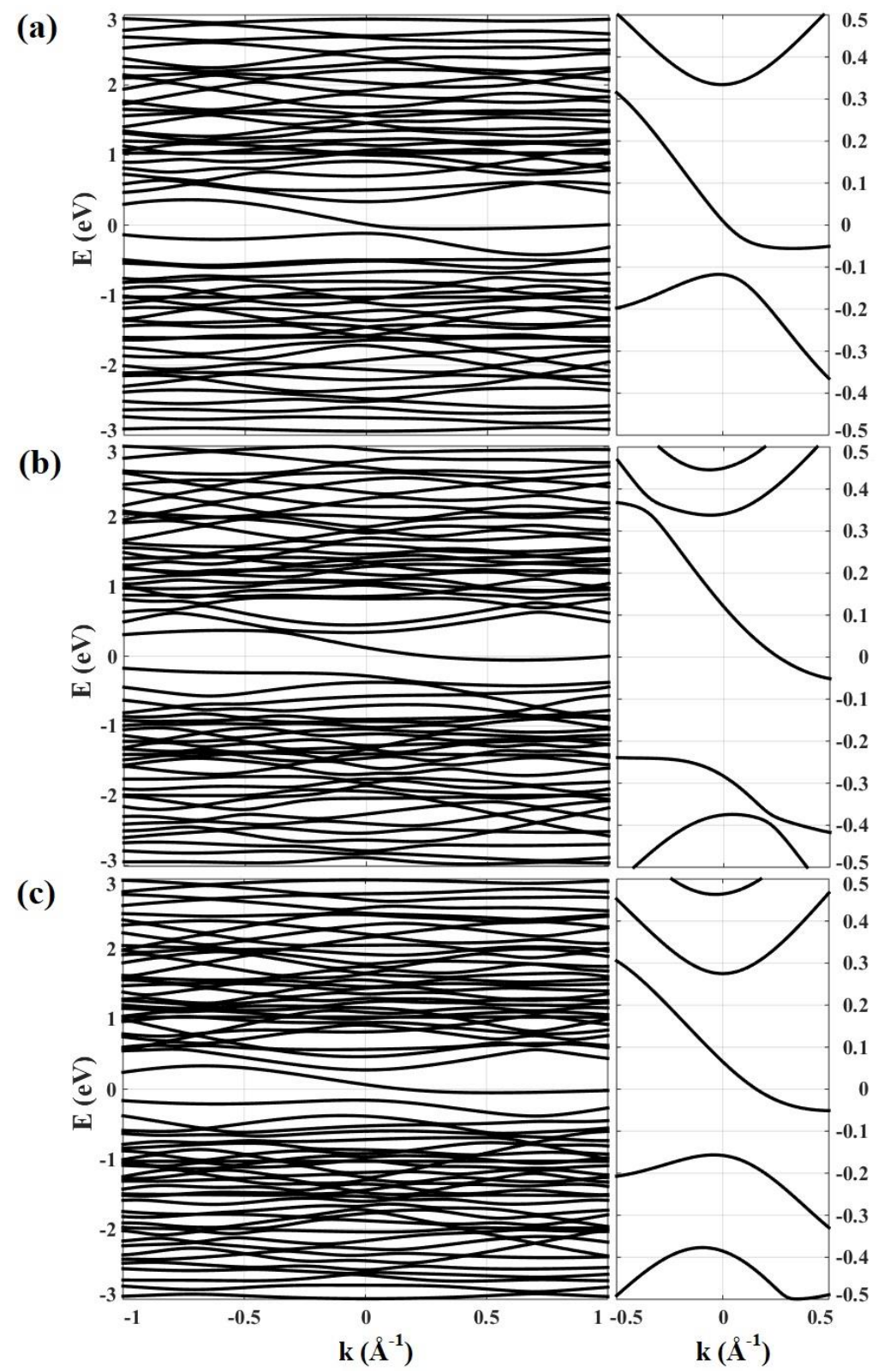

Figure 6. Energy band structure of defective AGeNR with (a) $N=3 p$, (b) $N=3 p+1$, (c) $N=3 p+2$.

\section{- The zigzag germanene nanoribbon (ZGeNR) energy band structure}

Figure 7a shows the unit cell of ZGeNR. The energy band structure of defect-free and defective ZGeNR are also indicated in figures $7 \mathrm{~b}$ and $7 \mathrm{c}$, respectively. It can be inferred from these results that ZGeNR in both cases have no energy band gap and metal behavior of nanoribbons is expected. 
(a)

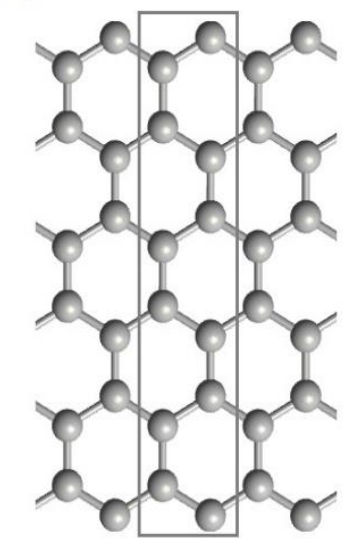

(b)

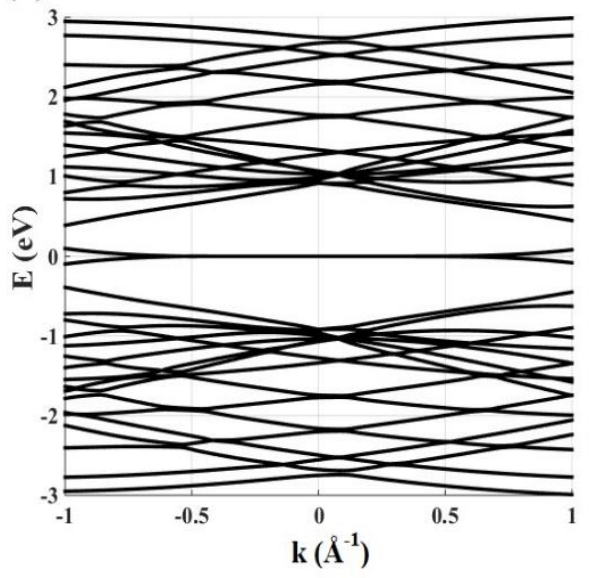

(c)

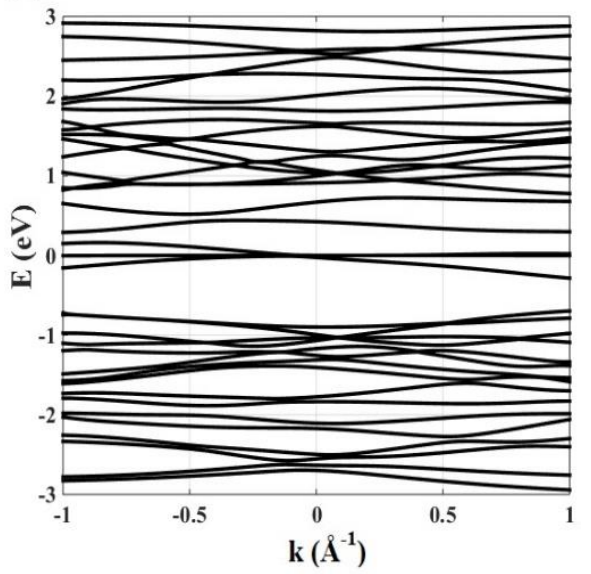

Figure 7. (a) Germanene nanoribbon in zigzag orientation, (b) energy band structure of defect-free, and (c) energy band structure of defective structures.

The obtained results of the present research demonstrate a rational agreement with previous theoretical outcomes

and experiments $[3,14,23]$.

\section{Conclusion}

Utilizing Tight-binding approximation method, the electronic properties of pristine and defective germanene are studied in this research. The impact of Stone-Wales defect on the electronic characteristics of infinite germanene monolayer as well as germanene nanoribbon in armchair and zigzag direction is analysed and the obtained results are compared. The results demonstrate the significant effect of Stone-Wales defect on the energy band structure of infinite and nanoribbon structure. The linearity and slope of E-k curves of germanene around the Dirac point are changed after applying the Stone-Wales defect, which results in a decrease in Fermi velocity and an increase in the band gap. While the armchair germanene nanoribbons with widths of $3 p$ and $3 p+1$ are semiconductor and the armchair germanene nanoribbon with width of $3 p+2$ shows the semi-metal behaviour, but after applying the StoneWales defect, the band gap of nanoribbons with widths of $3 p$ and $3 p+1$ are reduced, and is increased for width of $3 p+2$. In addition, there is no band gap in the energy band structure of zigzag germanene nanoribbon and the metallic behaviour is clearly visible.

\section{Declarations}

Funding: No Funding/ traditional publishing.

Conflicts of interest/Competing interests: The authors declare no competing interests.

Availability of data and material: The data that supports the findings of this study are available within the article and reference number.

Code availability: The results of software application are available within the article.

Authors' contributions: The main idea has been suggested by Dr. S. Mohammadi and K. Rahmani. The obtained results are also discussed and supported by Dr. S. Mohammadi and K. Rahmani.

Consent for publication: Traditional publishing. 


\section{References}

1- Rupp, C.J., Chakraborty, S., Ahuja, R., Baierle, R.J.: The effect of impurities in ultra-thin hydrogenated silicene and germanene: a first principles study. Phys. Chem. Chem. Phys. 27, 22210-22216 (2015)

2- Martins Ade, S., Veríssimo-Alves, M.: Group-IV nanosheets with vacancies: a tight-binding extended Hückel study. Journal of physics. Condensed Matter . 26, 365501 (2014)

3- Pang, Q., Zhang, Y., Zhang, J.M., Ji, V., Xu, K.W.: Electronic and magnetic properties of pristine and chemically functionalized germanene nanoribbons. Nanoscale, 3, 4330 (2011)

4- Chegel, R., Behzad, S.: Tunable electronic, optical, and thermal properties of two dimensional germanene via an external electric field. Scientific Reports 10, 1 (2020)

5- Acun, A., Zhang, L., Bampoulis, P., Farmanbar, M., Houselt, A.V., Rudenko, A.N., Lingenfelder, M., Brocks, G., Poelsema, B., Katsnelson, M.I., Zandvliet, H.J.W.: Germanene: the germanium analogue of graphene. J. Phys.: Condens. Matter. 27, 443002 (2015)

6- Ye, X.S., Shao, Z.G., Zhao, H., Yang, L., Wang, C.L.: Intrinsic carrier mobility of germanene is larger than graphene's: first-principle calculations. RSC Adv. 4, 21216 (2014)

7- Roome, N.J., Carey, J.D.: Beyond graphene: Stable elemental monolayers of silicene and germanene. ACS Appl. Mater. Interfaces. 6, 7743-7750 (2014)

8- Ren, C.C., Zhang, S.F., Ji, W.X., Zhang, C.W., Li, P., Wang, P.J.: Tunable electronic and topological properties of germanene by functional group modification. Nanomaterials. 8, 145 (2018)

9- Gupta, S., Singh, D., Rajput, K., Sonvane, Y.: Germanene: New electronic gas sensing material. RSC Advances. 104 (2016)

10- Wang, Y., Ji, W., Zhang, C., Li, S., Li, F., Li, P., Ren, M., Chen, X., Yuan, M., Wang, P.: Enhanced band gap opening in germanene by organic molecule adsorption. Materials Chemistry and Physics. 173, 379-384 (2016)

11- Wanga, X., Wu, Z.: Intrinsic magnetism and spontaneous band gap opening in bilayer silicene and germanene. Phys. Chem. Chem. Phys. 19, 2148-2152 (2017)

12- Ye, M., Quhe, R., Zheng, J., Ni, Z., Wang, Y., Yuan, Y., Tse, G., Shi, j., Gao, Z., Lu, J.: Tunable band gap in germanene by surface adsorption. Physica E Low-dimensional Systems and Nanostructures. DOI: 10.1016/j.physe.2013.12.016 (2013)

13- Zheng, j., Chi, F., Guo, Y.: Enhanced spin Seebeck effect in a germanene p-n junction. Journal of Applied Physics. 116, 243907 (2014)

14- Ni, Z., Liu, Q., Tang, k., Zheng, J., Zhou, J., Qin, R., Gao, Z., Yu, D., Lu, J.: Tunable band gap in silicene and germanene. Nano Lett. 12, 113-118 (2011)

15- Ali, M., Pi, X., Liu, Y., Yang, D.: Electronic and magnetic properties of graphene, silicene and germanene with varying vacancy concentration. AIP Advances. 7, 045308 (2017)

16- Jing, Y., Zhang, X., Wu, D., Zhao, X., Zhou, Z.: High carrier mobility and pronounced light absorption in methyl- terminated germanene: Insights from first-principles computations. J. Phys. Chem. Lett. 6, 42524258 (2015)

17- Kaloni, T.P., Schwingenschlogl, U.: Stability of germanene under tensile strain. Chemical Physics Letters, 583, 137-140 (2013)

18- Davila, M.E., Xian, L., Cahangirov, S., Rubio, A., Lay, G.L.: Germanene: a novel two-dimensional germanium allotrope akin to graphene and silicene. New Journal of Physics. 16, 095002 (2014)

19- Bishnoi, B., Ghoshb, b.: Spin transport in silicene and germanene. RSC Advances. 3, 26153-26159 (2013)

20- Tao, W., Kong, N., Ji, X., Zhang, Y., Sharma, A., Ouyang, J., Qi, B., Wang, J., Xie, N., Kang, C., Zhang, H., Farokhzad, O.C., Kim, J.S.: Emerging two-dimensional monoelemental materials (Xenes) for biomedical applications. Chem Soc Rev. 48, 2891-2912 (2019)

21- Dávila1, M.E., Lay, G.L.: Few layer epitaxial germanene: a novel two-dimensional Dirac material. Scientific Reports. 6, 20714 (2016)

22- Banerjee, L., Sengupta, A., Rahaman, H.: Carrier transport and thermoelectric properties of differently shaped germanene $(\mathrm{Ge})$ and silicene $(\mathrm{Si})$ nanoribbon interconnects. IEEE Transactions on Electron Devices. 66, 1 (2019)

23- Padilha, J.E., Pontes, R.B.: Electronic and transport properties of structural defects in monolayer germanene: An ab initio investigation. Solid State Communications. 225, 38-43 (2015) 
24- Ahmadi, M.T., Ismail, R., Anwar, S.: Handbook of research on nanoelectronic sensor modeling and applications, (IGI Global publisher, 2016)

25- Ismail, R., Ahmadi, M.T., Anwar, S.: Advanced nanoelectronics, (Boca Raton London New York: Taylor and Francis, 2012)

26- Foulkes, W.M.C.: Tight-Binding models and coulomb interactions for s, p, and d electrons., London SW7 2AZ United Kingdom, ISBN 978-3-95806-159-0 (2016)

27- McCann, E., Koshino, M.: The electronic properties of bilayer graphene. Reports on Progress in Physics. 76, 056503 (2013)

28- Nielsen, E., Rahman, R., Muller, R.: A many-electron tight binding method for the analysis of quantum dot systems. Journal of Applied Physics 112, 114304 (2012)

29- Seifert, G., Joswig, J.O.: Density-functional tight binding-an approximate density-functional theory method. Advanced Review, WIREs Comput Mol Sci. 2, 456-465 (2012)

30- Jiang, L., Marconcini, P., Sharafat Hossian, Md., Qiu, W., Evans, R., Macucci, M., Skafidas, E.: A tight binding and k.p study of monolayer stanene. Scientific Reports. 7, 12069 (2017) 
Figures

(a)

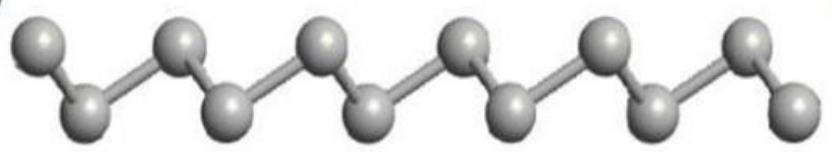

(b)

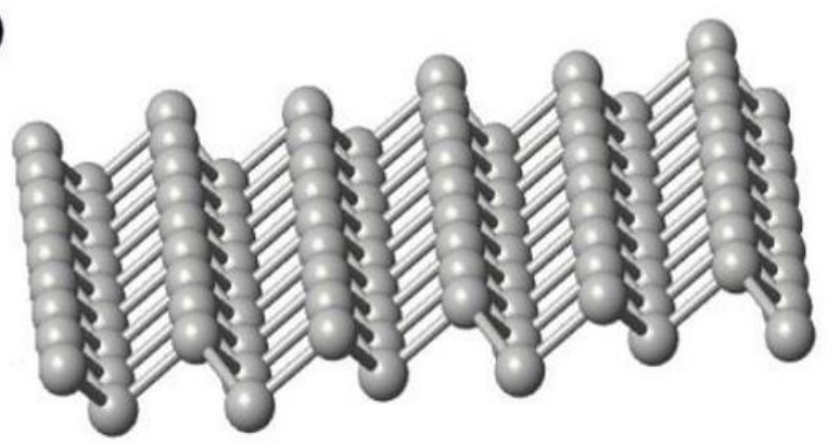

(c)

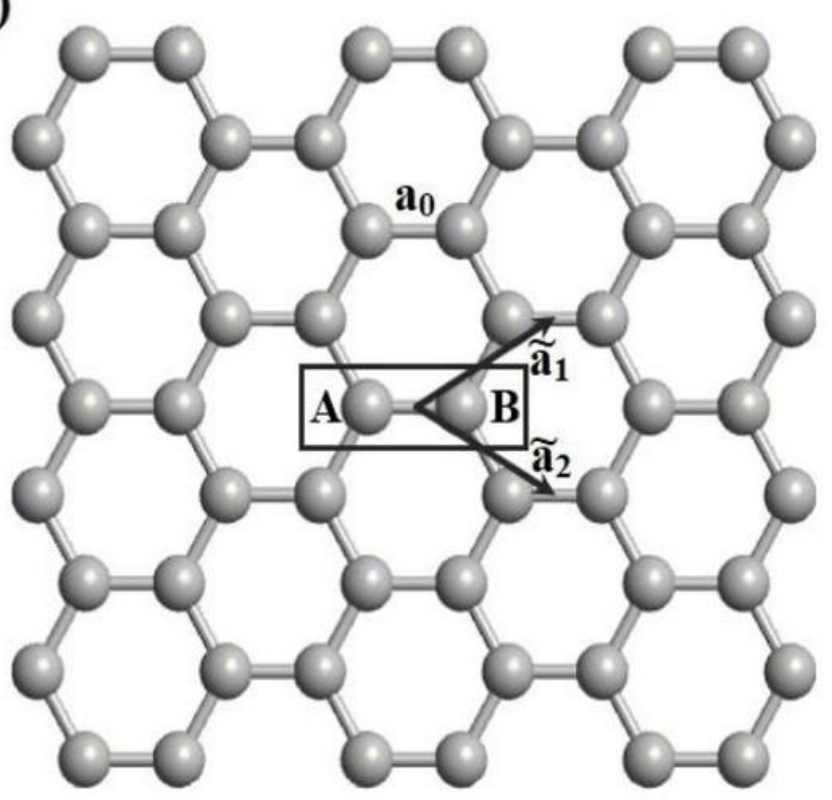

Figure 1

Structure of germanene in different views (a) and (b) side, and (c) top. ã 1 and ã2 are primitive lattice vectors, and $\mathrm{a} 0$ is the $\mathrm{Ge}-\mathrm{Ge}$ bond length. The unit cell includes two atoms, named $\mathrm{A}$ and $\mathrm{B}$. 


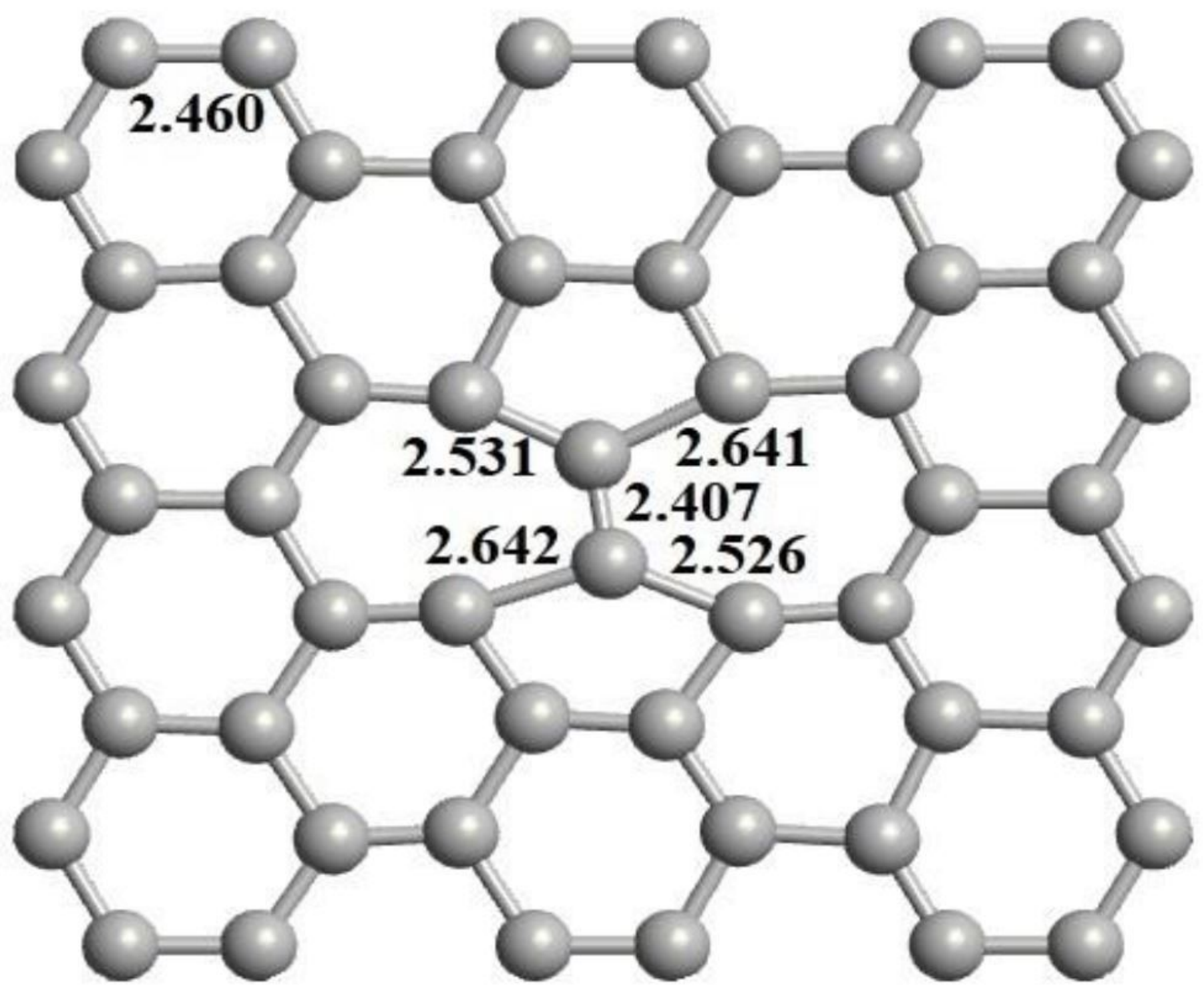

Figure 2

Structure of germanene monolayer with SW defect. 
(a)

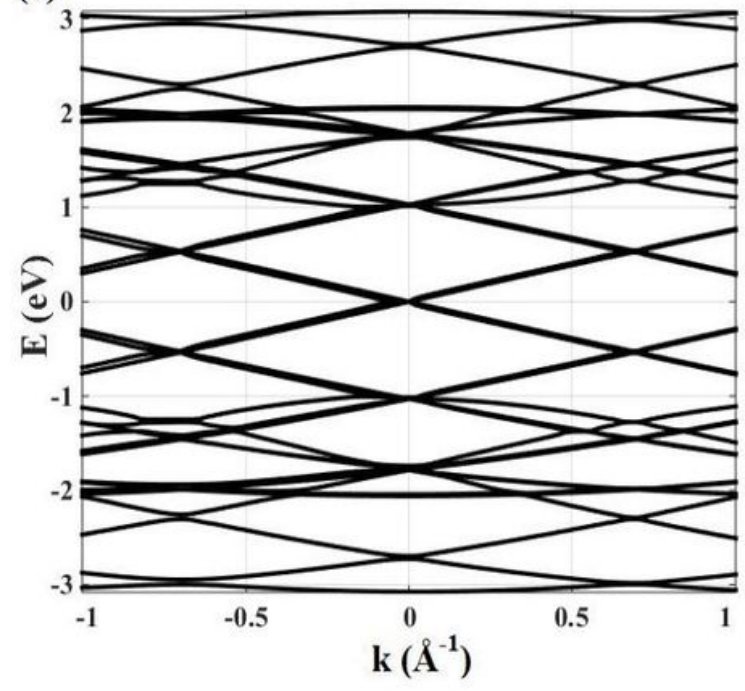

(b)

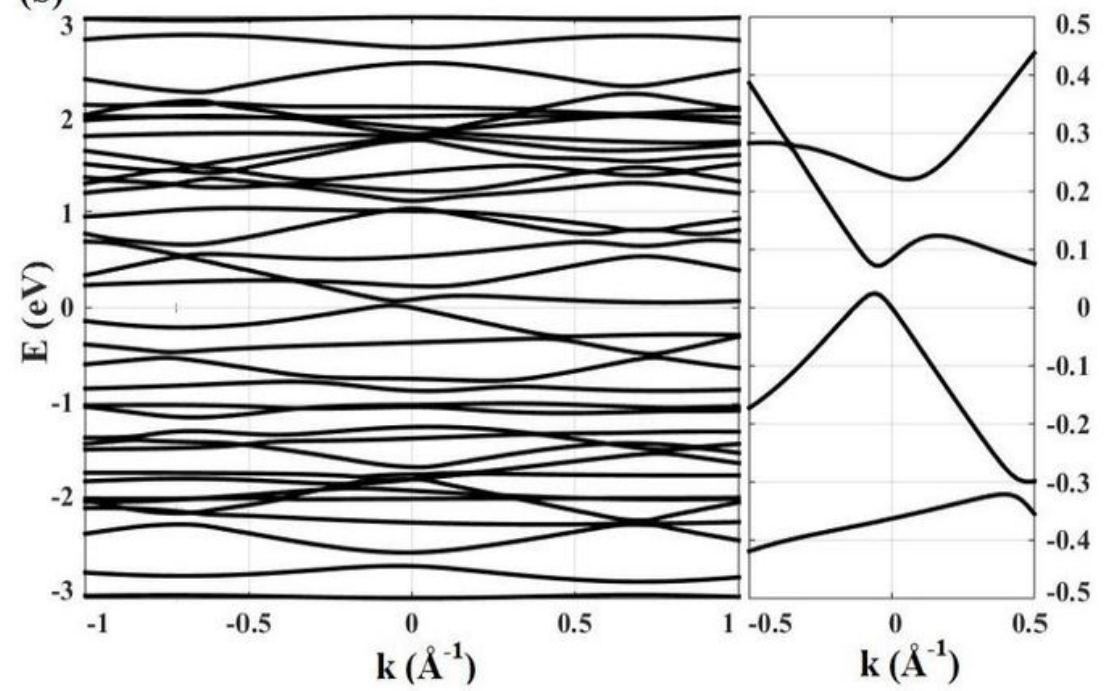

Figure 3

Energy band structure of (a) defect-free, and (b) defective infinite germanene sheet.

(a)

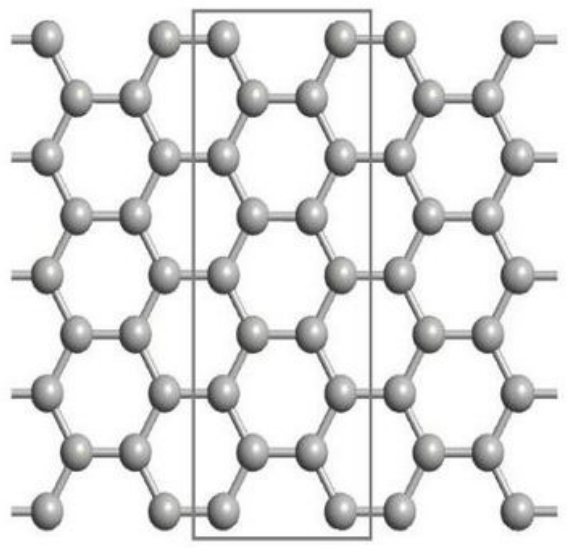

(b)

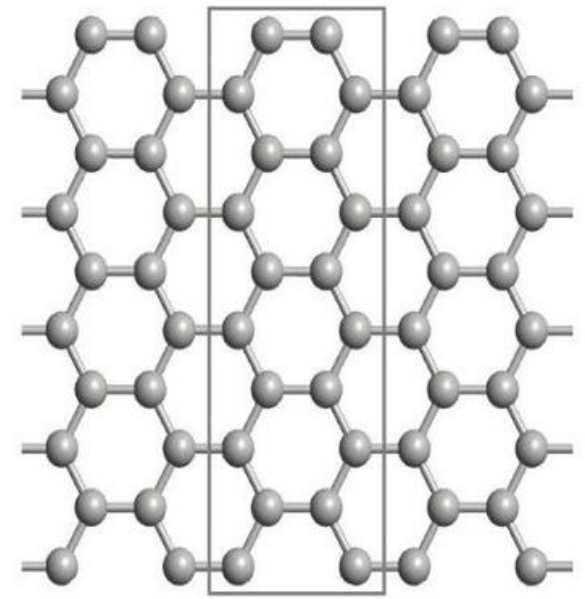

(c)

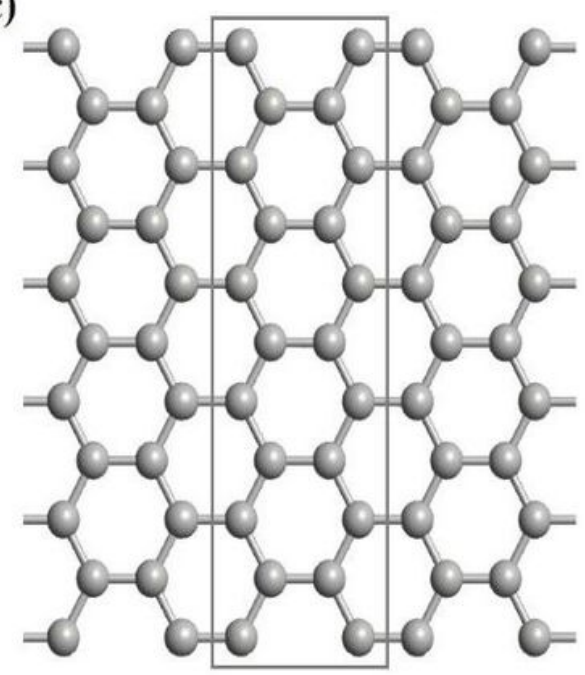

Figure 4

AGeNR with different nanoribbon widths, (a) 3p, (b) 3p+1, (c) 3p+2. 
(a)

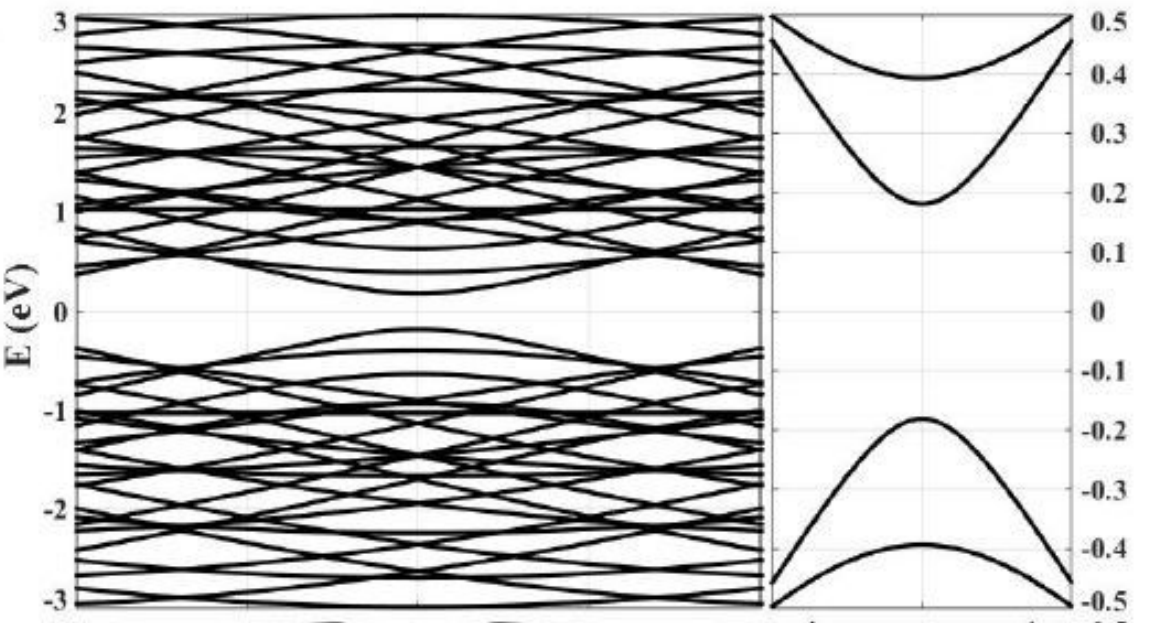

(b)

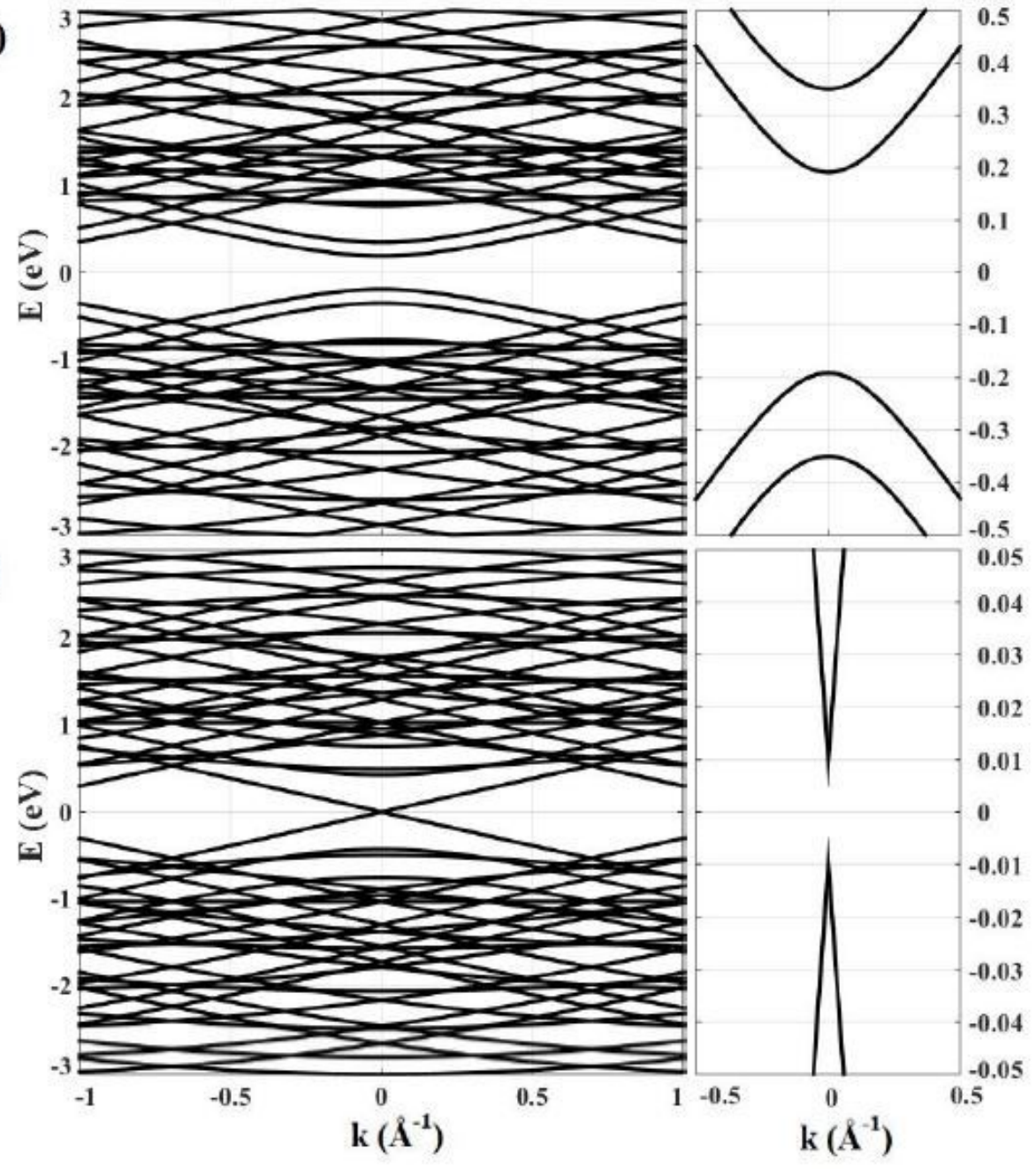

(c)

Figure 5

Energy band structure of defect-free AGeNR with (a) $N=3 p$, (b) $N=3 p+1$, (c) $N=3 p+2$. 
(a)

(b)
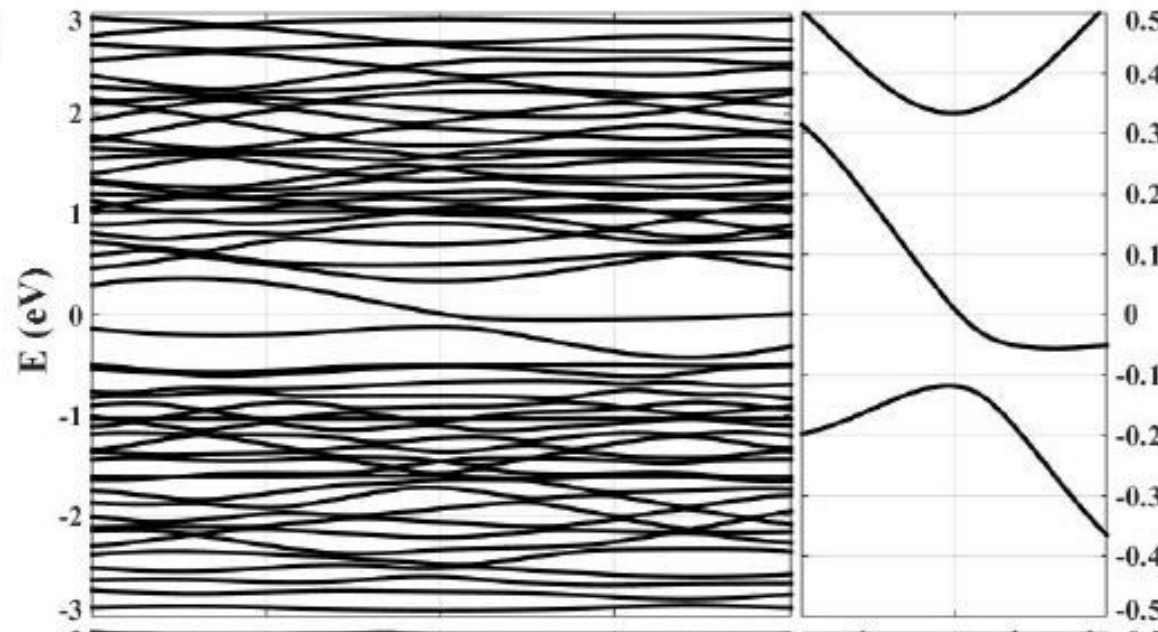

(c)
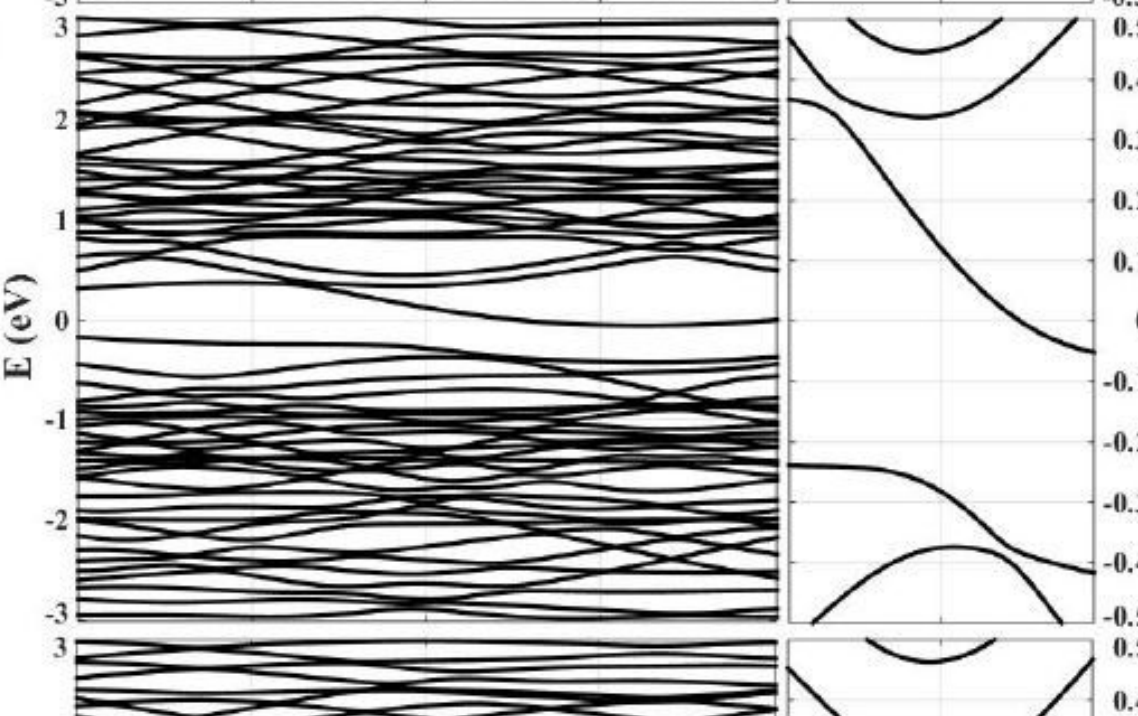

2
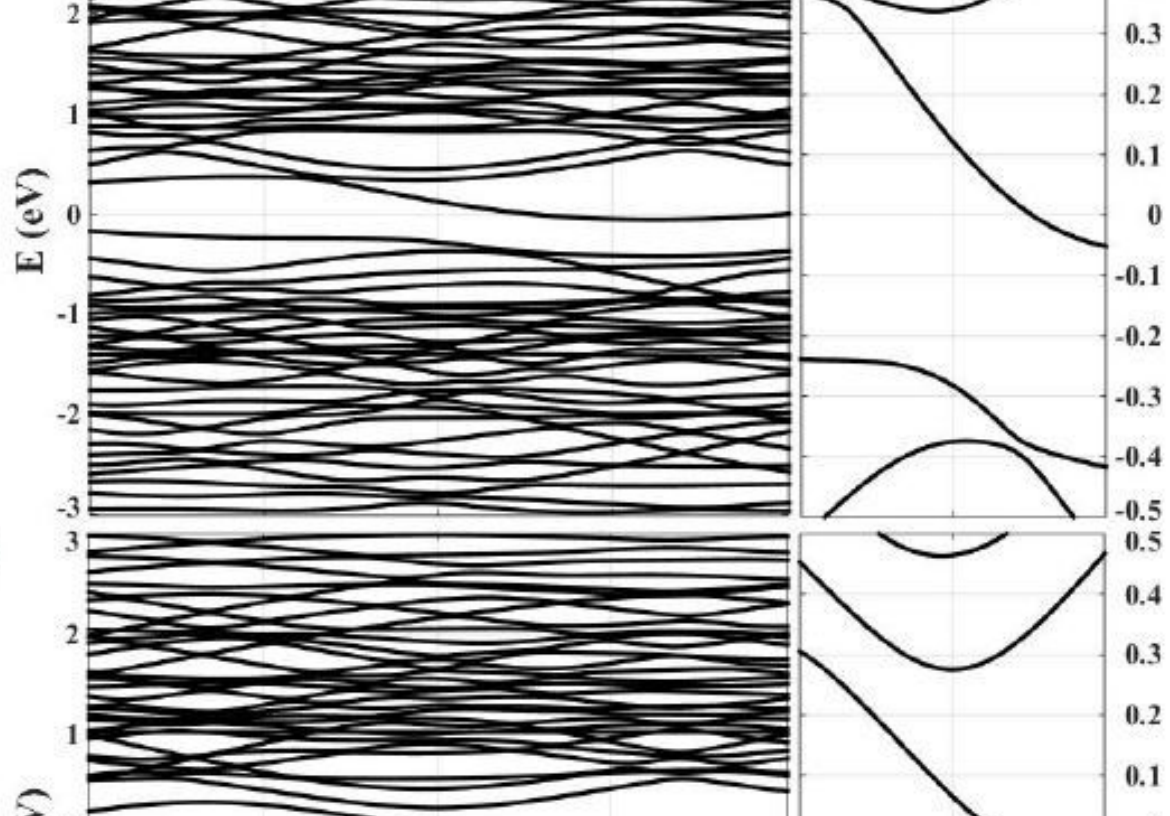

$\underset{1}{0}$

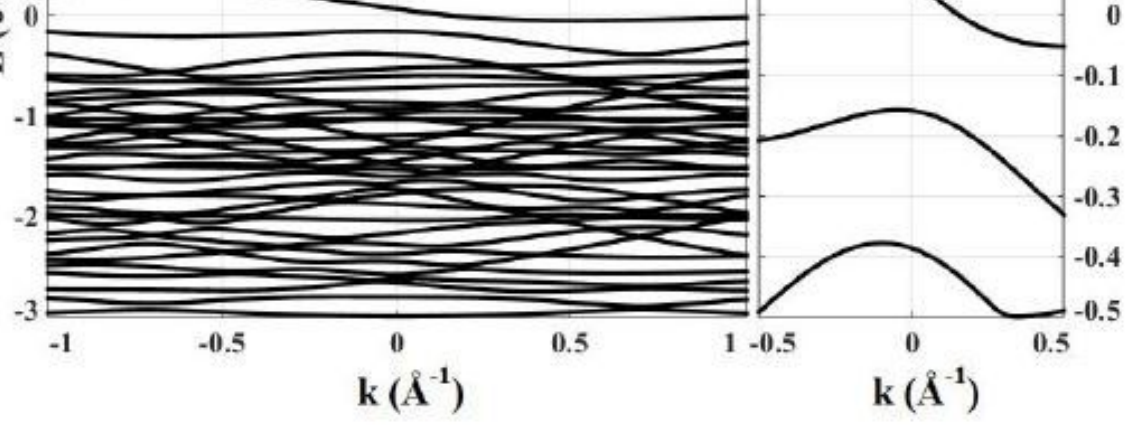

Figure 6

Energy band structure of defective AGeNR with (a) $N=3 p$, (b) $N=3 p+1$, (c) $N=3 p+2$. 
(a)

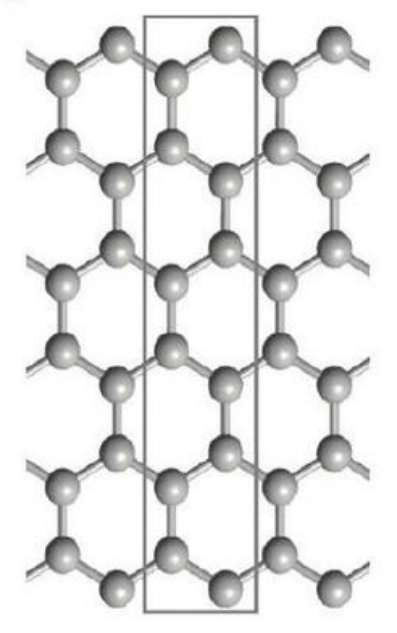

(b)

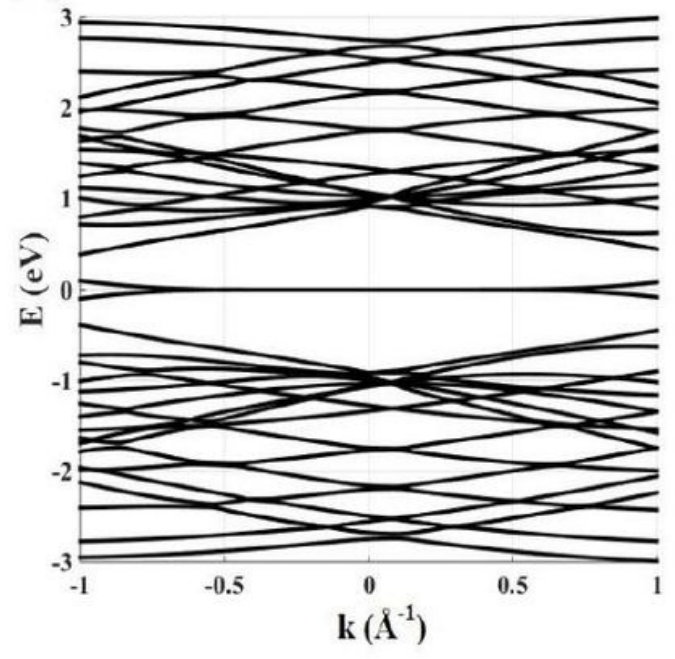

(c)

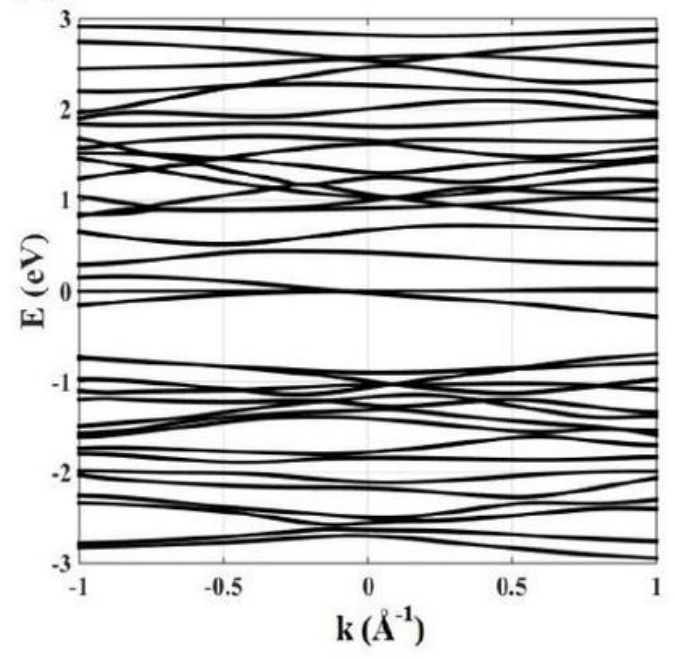

\section{Figure 7}

(a) Germanene nanoribbon in zigzag orientation, (b) energy band structure of defect-free, and (c) energy band structure of defective structures. 